\title{
Chloroquine enhances temozolomide cytotoxicity in malignant gliomas by blocking autophagy
}

\author{
Encouse B. Golden, M.D., Ph.D., ${ }^{1}$ Hee-Yeon Cho, Ph.D., ${ }^{2}$ Ardeshir Jahanian, Ph.D., ${ }^{4}$ \\ Florence M. Hofman, Ph.D., ${ }^{3}$ Stan G. Louie, Ph.D., ${ }^{5}$ Axel H. Schönthal, Ph.D., ${ }^{4}$ \\ and Thomas C. Chen, M.D., Ph.D., \\ ${ }^{1}$ Department of Radiation Oncology, New York University School of Medicine, New York, New York; and \\ Departments of ${ }^{2}$ Neurosurgery, ${ }^{3}$ Pathology, and ${ }^{4}$ Molecular Microbiology and Immunology, Keck School \\ of Medicine, and 5 Department of Pharmaceutical Sciences, School of Pharmacy, University of Southern \\ California, Los Angeles, California
}

\begin{abstract}
Object. In a recent clinical trial, patients with newly diagnosed glioblastoma multiforme benefited from chloroquine (CQ) in combination with conventional therapy (resection, temozolomide [TMZ], and radiation therapy). In the present study, the authors report the mechanism by which CQ enhances the therapeutic efficacy of TMZ to aid future studies aimed at improving this therapeutic regimen.

Methods. Using in vitro and in vivo experiments, the authors determined the mechanism by which CQ enhances TMZ cytotoxicity. They focused on the inhibition-of-autophagy mechanism of CQ by knockdown of the autophagyassociated proteins or treatment with autophagy inhibitors. This mechanism was tested using an in vivo model with subcutaneously implanted U87MG tumors from mice treated with CQ in combination with TMZ.

Results. Knockdown of the autophagy-associated proteins (GRP78 and Beclin) or treatment with the autophagy inhibitor, 3-methyl adenine (3-MA), blocked autophagosome formation and reduced CQ cytotoxicity, suggesting that autophagosome accumulation precedes CQ-induced cell death. In contrast, blocking autophagosome formation with knockdown of GRP78 or treatment with 3-MA enhanced TMZ cytotoxicity, suggesting that the autophagy pathway protects from TMZ-induced cytotoxicity. CQ in combination with TMZ significantly increased the amounts of LC3B-II (a marker for autophagosome levels), CHOP/GADD-153, and cleaved PARP (a marker for apoptosis) over those with untreated or individual drug-treated glioma cells. These molecular mechanisms seemed to take place in vivo as well. Subcutaneously implanted U87MG tumors from mice treated with CQ in combination with TMZ displayed higher levels of CHOP/GADD-153 than did untreated or individual drug-treated tumors.

Conclusions. Taken together, these results demonstrate that CQ blocks autophagy and triggers endoplasmic reticulum stress, thereby increasing the chemosensitivity of glioma cells to TMZ. (http://thejns.org/doi/abs/10.3171/2014.9.FOCUS14504)
\end{abstract}

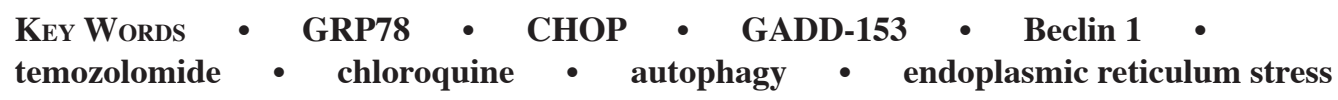

$\mathrm{I}$ $\mathrm{N}$ recent Phase I/II trials, chloroquine (CQ), a wellknown antimalarial agent, in combination with conventional therapies (resection, temozolomide [TMZ], and radiation therapy) significantly prolonged the median survival time in patients with newly diagnosed glioblastoma multiforme (GBM) from 11.4 to 25 months. ${ }^{4,5,39}$ Recently, the National Cancer Institute approved a Phase I/II clinical

\footnotetext{
Abbreviations used in this paper: $3-\mathrm{MA}=3$-methyl adenine; $\mathrm{BEC}$ = brain endothelial cell; BECN1 = Beclin 1 ; CFA = colony-formation assay; $\mathrm{CQ}=$ chloroquine; $\mathrm{ER}=$ endoplasmic reticulum; $\mathrm{GBM}$ = glioblastoma multiforme; PI3KC = class III phosphatidylinositol 3-kinase; PTEN = phosphatase and tensin homolog; si = small interfering; $\mathrm{TMZ}=$ temozolomide; $\mathrm{TMZ}^{\mathrm{R}}=\mathrm{TMZ}$ resistant; $\mathrm{TMZ}^{\mathrm{S}}=$ TMZ sensitive; TuBEC $=$ tumor-derived brain endothelial cell; UPR $=$ unfolded protein response.
}

trial to test the potential effects of hydroxychloroquine (a CQ analog) in combination with conventional therapy in patients with newly diagnosed GBM (ClinicalTrials.gov identifier, NCT00486603). Although the coordinating institutions for this clinical trial have already initiated enrollment of patients with GBM, the mechanism by which CQ acts to enhance the cytotoxic effects of TMZ has not been fully investigated. Thus, to better understand and improve this therapeutic regimen, we investigated the mechanism by which CQ acts to enhance TMZ cytotoxicity in glioma cells in vitro and in vivo.

Previous studies established that severe conditions within the GBM microenvironment results in the accumulation of endoplasmic reticulum (ER) protein aggregates, ER stress, and activation of the ER unfolded protein response (UPR) in glioma cells. ${ }^{20,35}$ Glioma cells protect 


\section{E. B. Golden et al.}

themselves from these conditions by overexpressing and rapidly inducing the production of the ER chaperone and cell survival protein GRP78/BiP. GRP78/BiP alleviates ER stress-associated apoptosis by folding ER protein, maintaining ER integrity, and preventing apoptosis. ${ }^{11,27}$ In addition to its aforementioned properties, GRP78 acts as an obligatory component of the autophagy pathway in mammalian cells. ${ }^{11,27,33}$ However, the cytoprotective role of GRP78-linked autophagy remains unclear.

Interestingly, several studies demonstrated a direct link between autophagy and ER stress. Autophagy countered the lethal effects of ER stress in tumor cells. ${ }^{2,15,27,43}$ In addition, recent reports established a link between the cytotoxic effects of TMZ, ER stress, and autophagy in glioma cells.$^{18,23}$ However, conflicting reports demonstrated autophagy as both tumor promoter and suppressor; thus, the exact relationship between autophagy, ER stress, and TMZ-induced cytotoxicity also remains unclear.

Chloroquine, a quinoline-based antimalarial, kills Plasmodium falciparum parasites in the intraerythrocytic stage by blocking acidic food vacuolar heme detoxification. ${ }^{38} \mathrm{CQ}$ at a physiological $\mathrm{pH}$ possesses the unique chemical property of being a weak base with the ability to easily traverse cellular lipid bilayers. However, once inside the acidic environment of $P$. falciparum food vacuoles, acidic molecules react with CQ and form an ionized CQ conjugate acid. CQ in the ionized form loses its ability to rapidly traverse vacuolar lipid bilayers and, hence, becomes trapped inside the acidic food vacuole.

Tumor cells contain acidic vacuoles known as lysosomes, which are comparable to the acidic food vacuoles of P.falciparum parasites. Acidic molecules in tumor cell lysosomes also react with CQ, thereby ionizing and trapping CQ in the lysosome. Upon reaching a critical concentration of CQ inside of the lysosome, CQ disrupts lysosomal enzymatic function and inhibits autophagosome clearance. $6,7,9$

Temozolomide, a DNA-alkylating agent, inhibits tumor growth by its ability to add methyl groups to the $\mathrm{O}^{6}$ position of guanines. . $^{3,12-14,40,41}$ In addition, TMZ induces autophagy..$^{18}$ Thus, it has been proposed that the autophagic pathway plays a role in the cytotoxic effects of TMZ. ${ }^{18,28}$

Because we previously demonstrated that GRP78/ $\mathrm{BiP}$ provides chemoresistance to TMZ in glioma cells, we investigated whether CQ, as an autophagy inhibitor, enhances TMZ cytotoxicity by blocking GRP78/BiPdependent autophagy. ${ }^{33,42}$ After testing our hypothesis, we found that blocking autophagy with CQ, 3-methyl adenine (3-MA), or knockdown of GRP78 enhanced the cytotoxic effects of TMZ. Importantly, we discovered that the cytotoxic effects of CQ alone were related directly to the presence of the ER chaperone GRP78/BiP, the autophagy-associated and $\mathrm{BH} 3$-only protein Beclin 1 (BECN1), the accumulation of autophagosomes and polyubiquitinated proteins, and the induction of the ER stress proapoptotic protein CCAAT enhancer-binding protein (C-EBP) homologous protein (CHOP/GADD-153). Last, we observed that the combination of TMZ and CQ synergistically triggered cell death via enhanced formation of LC3B-II (a marker of autophagosome levels), polyubiquitinated protein accumulation, $\mathrm{CHOP} / \mathrm{GADD}-153$ in- duction, and PARP (a marker of apoptosis) cleavage. We demonstrate here that autophagy protects glioma cells from TMZ cytotoxicity; however, blocking autophagy with CQ chemosensitizes glioma cells to TMZ.

\section{Methods}

Reagents

Chloroquine was obtained from Sigma Aldrich and dissolved in ultrapure water at a concentration of 100 $\mathrm{mM}$. Temozolomide (Temodar) was obtained from the University of Southern California Norris Cancer Hospital pharmacy and dissolved in dimethyl sulfoxide at a concentration of $50 \mathrm{mM}$ for our in vitro studies or suspended in phosphate-buffered saline at $2 \mathrm{mg} / \mathrm{ml}$ for our in vivo studies.

\section{Cell Lines and Culturing}

Human glioblastoma cell lines (LN229 and U251) were kindly supplied by Dr. Frank Furnari. The human glioblastoma cell line U87MG was purchased from the American Tissue Culture Collection. All glioma cell lines were propagated in DMEM (Cellgro) supplemented with $10 \%$ fetal bovine serum, $100 \mathrm{U} / \mathrm{ml}$ penicillin, and $0.1 \mathrm{mg} /$ $\mathrm{ml}$ streptomycin in a humidified incubator at $37^{\circ} \mathrm{C}$ and a $5 \% \mathrm{CO}_{2}$ atmosphere. TMZ-resistant $\left(\mathrm{TMZ}^{\mathrm{R}}\right)$ cells lines were developed from the surviving clones treated with $100 \mu \mathrm{M}$ TMZ for 1 month. Human tumor-derived brain endothelial cells (TuBECs) and human brain endothelial cells (BECs) were isolated within University of Southern California Institutional Review Board guidelines and were seeded and propagated as described in detail elsewhere. ${ }^{42}$

\section{MTT Assay}

Forty-eight-hour MTT assays were performed in 96well plates with the use of $3.0 \times 10^{3}$ cells per well for the glioblastoma cell lines described in detail elsewhere. ${ }^{21}$ In individual experiments, each treatment condition was set up in quadruplicate, and each experiment was repeated 1-5 times independently.

\section{Colony-Formation Assay}

U251 or LN229 cells were seeded into 6-well plates at 200 cells per well. After complete cell adherence, the cells were exposed to drug treatment (in triplicate) for 48 hours. Thereafter, the drug was removed, fresh growth medium was added, and the cells were kept in culture undisturbed for 12-14 days, during which time the surviving cells spawned a colony of proliferating cells. The colonies were visualized by staining for 4 hours with $1 \%$ methylene blue (in methanol) and then counted.

\section{Immunoblots and Antibodies}

Preparation of cell lysates and determination of protein concentrations were performed as described previously. ${ }^{21}$ Fifty micrograms of lysate from each sample was run in parallel. The primary antibodies were purchased from Cell Signaling Technologies or Santa Cruz Biotechnology, Inc., and used according to the manufacturers' instructions. The blocking buffer and fluorescent-conjugated secondary an- 
tibodies were purchased from Li-Cor Biosciences and used according to protocols supplied by the manufacturer. The membranes were scanned and analyzed with the Odyssey infrared imaging system (Li-Cor Biosciences) according to the manufacturer's instructions. Each immunoblot assay was repeated at least once.

\section{Drug Treatment of Nude Mice}

All animal protocols were approved by the University of Southern California Institutional Animal Care and Use Committee, and all applicable policies were strictly observed during the course of this study. Four- to 6-weekold male athymic nu/nu mice were obtained from Harlan Laboratories and implanted subcutaneously with $5 \times 10^{6}$ U87MG glioma cells. Once tumors of $\sim 300 \mathrm{~mm}^{3}$ had developed, the mice received drug treatments. TMZ and CQ were given as a daily oral gavage with a stainless steel ball-head feeding needle (Popper and Sons, Inc.). After a total of 48 hours, the mice were killed, and tumors were collected for analysis. The mice were closely monitored with regard to body weight, food consumption, and clinical signs of toxicity; no differences between non-drugtreated control animals and drug-treated animals were detected within these parameters.

\section{Immunohistochemical Staining}

Immunohistochemical staining was performed on 7- $\mu$ m-thick frozen-tissue sections on glass slides (VWR) with antibody to $\mathrm{CHOP} / \mathrm{GADD}-153$ (Santa Cruz Biotechnology) and with the use of the Vectastain avidinbiotin complex kit (Vector Laboratories) used in accordance with the manufacturers' recommendations and as described elsewhere. ${ }^{32}$ The stained slides were mounted on glass coverslips, sealed with nail polish, and analyzed under the microscope.

\section{Results}

\section{Chloroquine Enhances the Cytotoxic Effects of} Temozolomide

To determine the role of autophagy in relation to the cytotoxic effects of TMZ, we treated glioma cells with TMZ in the presence or absence of CQ in a series of colony-formation assays (CFAs) (Fig. 1A). After treating U251 (p53 mutant, phosphatase and tensin homolog [PTEN] mutant) and LN229 (p53 mutant, PTEN wild type) glioma cell lines with the autophagy inhibitor $C Q$, we observed that the percentages of colonies formed relative to those formed by the untreated control were reduced by $\sim 40 \%$ and $\sim 30 \%$, respectively. ${ }^{17,36,37}$ Furthermore, when we treated U251 and LN229 glioma cells with $20 \mu \mathrm{M}$ TMZ, TMZ reduced the percentages of colonies formed by $\sim 30 \%$ and $\sim 60 \%$, respectively. When we treated U251 and LN229 glioma cells with the combined therapy of TMZ and CQ, the combined regimen reduced the percentage of colonies formed by $\sim 85 \%$ and $\sim 97.5 \%$, respectively. It is notable that the combined effect of CQ and TMZ in glioma cell lines was greater than the additive cytotoxic effects of each therapy alone. CQ in combination with TMZ resulted in a calculated combination index of $<1$; thus, CQ synergistically enhanced the cytotoxic effects of TMZ in CFAs. ${ }^{44}$
To better understand the mechanism of action of the synergistic cytotoxic effects of TMZ and CQ in glioma cells, we treated small interfering (si)RNA-transfected U251 cells with TMZ and CQ in a series of CFAs. We initially transfected U251 cells with siRNA to a scrambled sequence (siControl), GRP78/BiP, BECN1, or CHOP/ GADD-153. After transfection, we treated the cells with CQ, TMZ, or CQ in combination with TMZ and determined the cytotoxicities via CFAs (Fig. 1B and C). We found that treatment of siControl-transfected U251 cells with $20 \mu \mathrm{M} \mathrm{TMZ}$ reduced the percentage of colonies formed by $\sim 50 \%$. Knockdown of the ER chaperone GRP78 with siGRP78, however, enhanced the cytotoxic effects of TMZ by further reducing the percentage of colonies formed by an additional $\sim 10 \%$. On the other hand, attenuation of CHOP/GADD-153 induction with siCHOP mitigated the cytotoxic effects of TMZ by $\sim 10 \%$.

Treatment of siControl-transfected U251 cells with $10 \mu \mathrm{M}$ CQ reduced the percentage of colonies formed by $\sim 70 \%$. Knockdown of GRP78 with siGRP78 blunted the cytotoxic effects of CQ by $\sim 10 \%$. Likewise, knockdown of the autophagy-linked protein BECN1 with siBECN1 diminished the cytotoxic effects of CQ by $\sim 10 \%$. In addition, the attenuation of CHOP/GADD-153 induction with siCHOP lessened the cytotoxic effects of CQ by $\sim 35 \%$.

Treatment of siControl-transfected U251 cells with $20 \mu \mathrm{M}$ TMZ in the presence of $10 \mu \mathrm{M}$ CQ reduced the percentage of colonies formed by $\sim 95 \%$. Knockdown of GRP78 or BECN1 in U251 cells had little to no effect on the combined regimen of TMZ and CQ. In contrast, siCHOP protected glioma cells from the cytotoxic effects of the combined regimen of TMZ and CQ by $35 \%$. Thus, the cytotoxic effects of TMZ in combination with CQ act via the induction of the apoptotic protein CHOP/GADD-153.

To confirm the specificity and effectiveness of our siRNAs to GRP78/BiP, BECN1, and CHOP/GADD-153 mRNAs, we assayed for their respective protein expression levels in a Western blot analysis (Fig. 1B and C). In comparison with siControl, siGRP78 and siBECN1 mitigated the expression of the constitutively expressed proteins GRP78 and BECN1, respectively. It is interesting to note that knockdown of GRP78, but not BECN1, triggered CHOP/GADD-153 induction and PARP cleavage. Similarly, $50 \mu \mathrm{M}$ CQ triggered CHOP/GADD-153 induction and PARP cleavage in siControl-transfected cells. However, $50 \mu \mathrm{M}$ CQ-treated U251 cells transfected with siCHOP exhibited an attenuation of CHOP/GADD153 induction and PARP cleavage.

In summary, the results of the CFA and the Western blot assay (Fig. 1A-C) reveal several key mechanistic features about TMZ and CQ. CQ, a known inhibitor of autophagy clearance, enhances the cytotoxic effects of TMZ, a known autophagy inducer. ${ }^{18}$ GRP78 maintains ER integrity and assists in autophagosome formation independent of BECN1-dependent autophagy. ${ }^{27}$ Preventing GRP78-dependent autophagy by downregulating GRP78 enhances the cytotoxic effects of TMZ but protects from the cytotoxic effects of CQ. The attenuation of $\mathrm{CHOP} /$ GADD-153 induction mitigates the cytotoxic effects of TMZ and CQ individually and in combination. In addition, blocking BECN1-dependent autophagy by down- 
E. B. Golden et al.
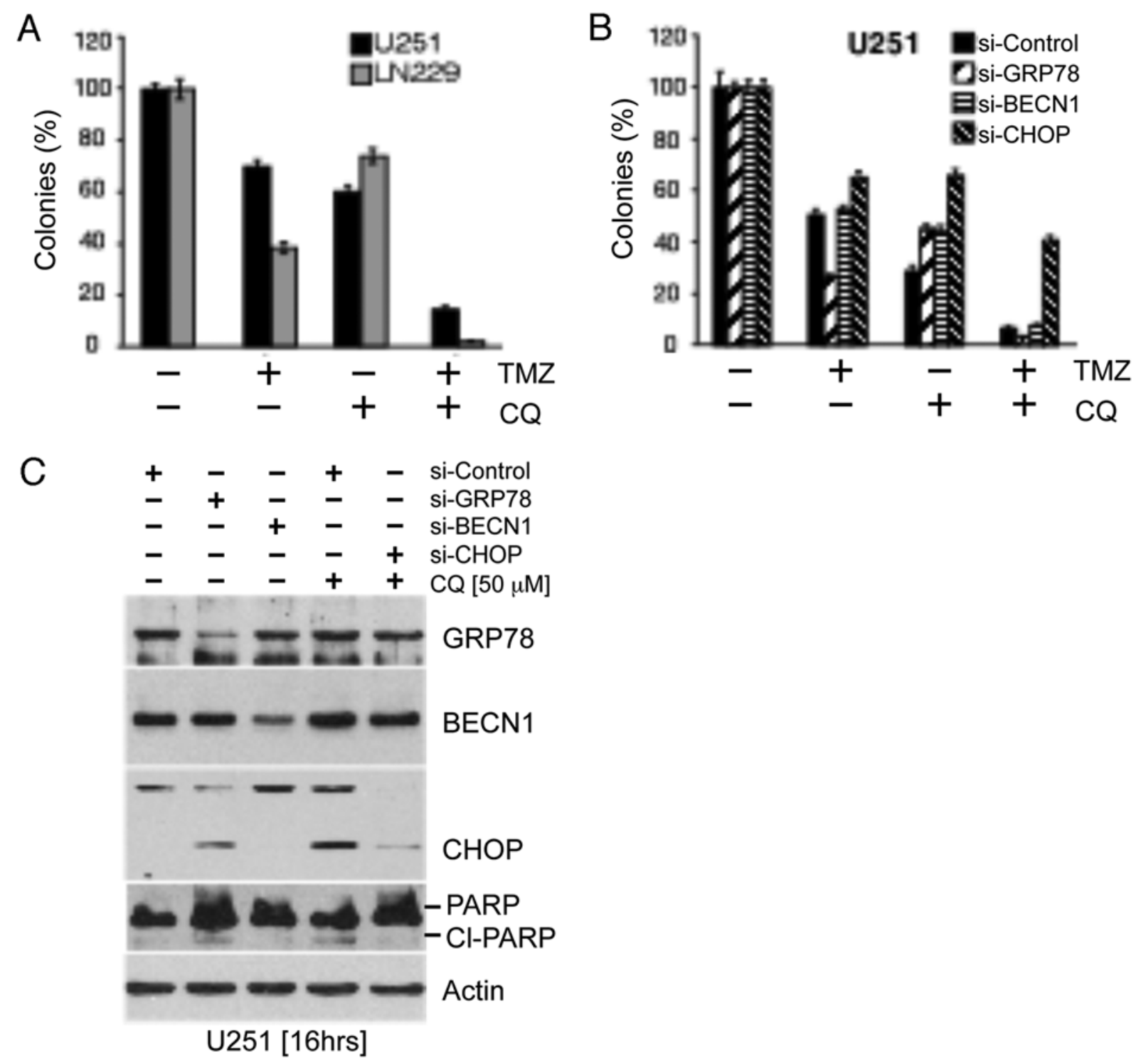

FIG. 1. Chloroquine enhances the cytotoxic effects of TMZ. A: U251 or LN229 cells ( 200 cells/well) were seeded in 6 -well plates and were left untreated or treated with $20 \mu \mathrm{M}$ TMZ or CQ (U251, $10 \mu \mathrm{M} \mathrm{CQ;} \mathrm{LN229,} 20 \mu \mathrm{M}$ CQ) alone or in combination for 48 hours, and the numbers of long-term surviving cells that were able to spawn colonies were determined 2 weeks thereafter (CFA). U251 cells were transiently transfected with siRNA directed to GRP78, BECN1, or CHOP/GADD-153. A scrambled siRNA sequence (si-Control) was used as a transfection control. Twenty-four hours after transfection, cells were seeded in parallel for either a CFA (B) or Western blot analysis (C). The cells that were seeded for a CFA were left untreated or treated with $20 \mu \mathrm{M}$ TMZ, $10 \mu \mathrm{M} C Q$, or the combination of TMZ and CQ. Thereafter, the drug was removed, fresh growth medium was added, and the cells were kept in culture undisturbed for 12-14 days, during which time the surviving cells spawned colonies of proliferating cells. The colonies were visualized by staining for 4 hours with $1 \%$ methylene blue (in methanol) and then counted. The columns represent the mean percentages of surviving cells from triplicate experiments; bars indicate the SD. The number of colonies obtained from the untreated controls was set at $100 \%$. The cells that were seeded for Western blot analysis were either left untreated or treated with $50 \mu \mathrm{M} \mathrm{CQ}$ for 16 hours. C: To confirm the knockdown of targeted proteins, we analyzed the protein levels of GRP78, BECN1, CHOP/GADD-153, and PARP via Western blot analysis. $\beta$-Actin (Actin) was used as a loading control. cl-PARP = cleaved PARP.

regulating $\mathrm{BECN} 1$ does not induce either $\mathrm{CHOP} / \mathrm{GADD}-$ 153 or PARP cleavage. However, it does protect from the cytotoxic effects of CQ but not TMZ. Therefore, CQ may enhance the cytotoxic effects of TMZ by blocking GRP78-dependent autophagy and inducing the expression of the proapoptotic protein CHOP/GADD-153.
Chloroquine Blocks GRP78-Dependent Autophagy Clearance

To investigate the mechanistic details of the relationship between CQ and GRP78-dependent autophagy, we assayed for the protein levels responsible for autophagosome accumulation and ER stress-induced apoptosis 


\section{Chloroquine enhances temozolomide therapy}

via Western blot analysis (Fig. 2 upper). It is important to note that we assayed for the presence of the free $\mathrm{B}$ isoform of microtubule-associated protein light-chain 3 (LC3B-I) and its autophagosomal membrane-bound phosphatidylethanolamine conjugate (LC3B-II) and interpreted our results on the basis of previous reports. ${ }^{8,22,30}$ We interpreted the presence of LC3B-I as the amount of LC3B-I readily available for conversion to LC3B-II. Moreover, we interpreted the levels of LC3B-II as a direct correlate to autophagosome levels; thus, when we block autophagosome clearance with $\mathrm{CQ}$, the ratio of LC3B-I to LC3B-II inversely indicates the efficiency of autophagosome formation and flux through the autophagy system..$^{8,22,30}$ In addition to LC3B-I and LC3B-II, we assayed
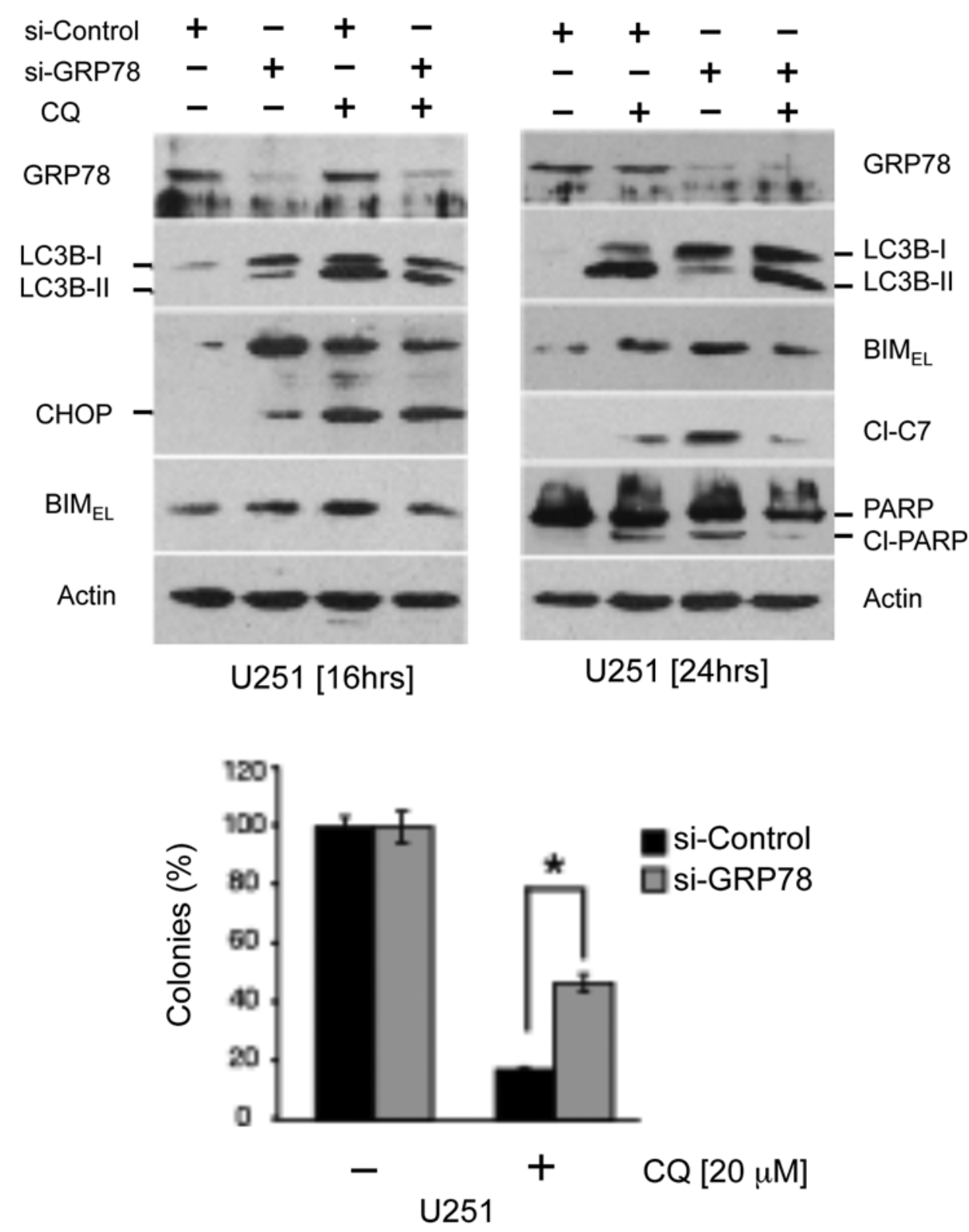

FIG. 2. Chloroquine blocks GRP78-dependent autophagy. U251 cells were transiently transfected with siRNA directed at GRP78 (si-GRP78). Scrambled siRNA directed at a nonspecific target was used as a transfection control (si-Control). Twentyfour hours after transfection, parallel cultures were seeded for either Western blot analysis or a CFA. Cells that were seeded for Western blot analysis were either untreated or treated with $50 \mu \mathrm{M} \mathrm{CQ}$ for 16 or 24 hours. Upper: The expression levels

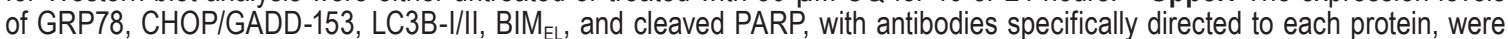
analyzed. $\beta$-Actin (Actin) was used as a loading control. Lower: Cells that were seeded for a CFA were either untreated or treated with $20 \mu \mathrm{M} \mathrm{CQ}$ for 48 hours. Thereafter, the drug was removed, fresh growth medium was added, and the cells were kept in culture and undisturbed for 12-14 days, during which time the surviving cells spawned a colony of proliferating cells. Colonies were visualized by staining for 4 hours with $1 \%$ methylene blue (in methanol) and then counted. For the CFAs, the columns represent the mean percentage of surviving cells from triplicate experiments; bars indicate the SD. The number of colonies obtained from the untreated controls was set at $100 \%$. The asterisk indicates significance $(p<0.05)$. 
for induction of the proapoptotic proteins CHOP/GADD153 and $\mathrm{Bcl} 2$ interacting mediator of cell death $\left(\mathrm{BIM}_{\mathrm{EL}}\right)$ and cleavage of caspase 7 and PARP.

We found that knockdown of GRP78 alone in U251 cells induced LC3B-I and resulted in a modest amount of LC3B-II conversion (ratio of LC3B-I/LC3B-II > 1) (Fig. 2 upper). Similar to knocking down GRP78, treatment of siControl-transfected U251 cells with $50 \mu \mathrm{M}$ CQ for 16 and 24 hours also increased LC3B-I levels (Fig. 2 upper); however, treatment with $\mathrm{CQ}$ resulted in a much greater increase of LC3B-II levels and conversion (LC3B-I/ LC3B-II < 1). It is interesting to note that knockdown of GRP78 in U251 cells attenuated the ability of CQ to increase LC3B-II levels (Fig. 2 upper left) and conversion (LC3B-I/LC3B-II $\approx 1$ ) (Fig. 2 upper), suggesting that knockdown of GRP78 in CQ-treated cells reduced the rate of autophagosome formation and accumulation.

To further delineate the mechanistic details of CQ and GRP78-dependent autophagy, we also assayed markers of ER stress and apoptosis (Fig. 2 upper). We found that GRP78 knockdown not only diminished the ability of CQ to convert LC3B-I to LC3B-II but also limited its ability to induce CHOP/GADD-153 and $\mathrm{BIM}_{\mathrm{EL}}$ and to cause cleavage of caspase 7 and PARP (Fig. 2 upper). Thus, reducing GRP78-dependent autophagy diminished the flux through the autophagic system and mitigated the ability of CQ to cause autophagosome accumulation and apoptosis.

To confirm that blocking GRP78-dependent autophagy prevents the cytotoxic effects of $\mathrm{CQ}$, we further investigated the cytotoxic effects of the combination of siGRP78 and CQ in a CFA (Fig. 2 lower). In a CFA, the treatment of siControl-transfected cells with $20 \mu \mathrm{M}$ CQ resulted in a reduction of the percentage of colonies formed by $\sim 85 \%$. However, treatment of siGRP78-transfected cells with $20 \mu \mathrm{M}$ CQ reduced the percentage of colonies formed by only $\sim 65 \%$. Thus, knockdown of GRP78 attenuated the cytotoxic effects of CQ in a CFA by 20\%.

\section{GRP78-Dependent Autophagy Modulates Temozolomide Cytotoxicity}

Autophagy was reported previously to play a role in TMZ cytotoxicity. ${ }^{18}$ However, because autophagy can serve as either a cytoprotective or a cytotoxic mechanism in tumor cells, the role of autophagy in TMZ-treated glioma cells remains controversial. Thus, we blocked various steps along the autophagy pathway to investigate whether autophagy serves to enhance or protect from the cytotoxic effects of TMZ in glioma cells.

To determine the role of autophagy in TMZ-treated glioma cells, we treated glioma cells in a CFA with TMZ or CQ in the presence or absence of the autophagy inhibitor 3-MA. 3-MA specifically inhibits the ability of class III phosphatidylinositol 3-kinase (PI3KC) to form an autophagosome initiation complex with BECN1, thereby preventing autophagosome formation. ${ }^{1}$ Moreover, 3-MA inhibits the ER UPR by suppressing the activation of the ER stress transducers PERK, IRE1, and ATF-6 and their downstream targets GRP78, ATF-4, CHOP/GADD-153, EIF- $2 \alpha$, JNK, and XBP- 1.27

It is interesting to note that when U251 cells were treated with $1 \mathrm{mM} 3-\mathrm{MA}$ in combination with $20 \mu \mathrm{M}$ TMZ, 3-MA significantly enhanced the cytotoxic effects of TMZ. In contrast, when U251 cells were treated with 1 mM 3-MA in combination with $20 \mu \mathrm{M} \mathrm{CQ}, 3-\mathrm{MA}$ significantly reduced the cytotoxic effects of CQ. Because CQ blocks autophagosome clearance and knockdown of either BECN1 or GRP78 and treatment with 3-MA diminished the cytotoxic effects of CQ, CQ may exert its cytotoxic effects by blocking both the PI3KC3-BECN1-dependent and GRP78-dependent autophagy pathways (Figs. 1B and C, 2, and 3). Nevertheless, because knockdown of GRP78 (but not BECN1) or treatment with 3-MA enhanced the cytotoxic effects of TMZ, the rate of GRP78-dependent autophagosome formation may inversely modulate TMZ cytotoxicity (Figs. 1B and C, and 3). These data, taken together, indicate that CQ is most effective when the rate of autophagosome production is elevated. TMZ cytotoxicity, on the other hand, is reduced as a consequence of autophagosome production. Thus, when CQ is combined with TMZ, CQ effectively kills glioma cells that would have otherwise survived in the presence of TMZ alone.

\section{Temozolomide-Resistant Cells Are Sensitive to Chloroquine}

Although TMZ increases the mean probability of survival in patients with GBM, glioma cells eventually develop chemoresistance to TMZ, which results in tumor recurrence and eventual death of the patient with GBM. To determine whether $\mathrm{TMZ}^{\mathrm{R}}$ glioma cells remained sensitive to $\mathrm{CQ}$, we treated $\mathrm{TMZ} \mathrm{Z}^{\mathrm{R}}$ glioma cell lines and their parental TMZ-sensitive $\left(\mathrm{TMZ}^{\mathrm{S}}\right)$ cell lines with increasing concentrations of CQ in a series of MTT assays (Fig. $4 \mathrm{~A}-\mathrm{C})$.

It was surprising that when we treated $\mathrm{TMZ}^{\mathrm{S}}$ and

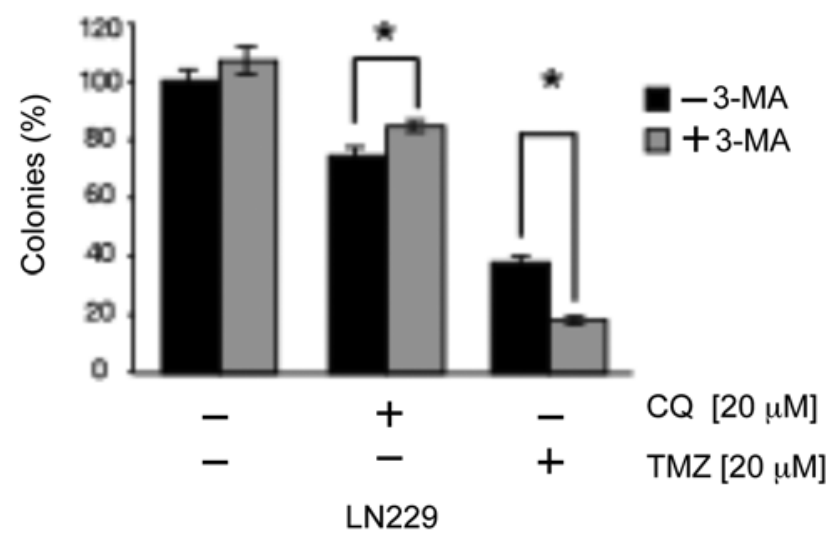

FIG. 3. 3-methyl adenine attenuates $C Q$ cytotoxicity but enhances TMZ cytotoxicity. U251 cells (200 cells/well) were seeded into 6-well plates and allowed to attach to the plates overnight. Thereafter, the cells were either untreated or treated with $20 \mu \mathrm{M} \mathrm{CQ}$ or $20 \mu \mathrm{M} \mathrm{TMZ}$ in the presence or absence of 3-MA for 48 hours. The drug was removed, fresh growth medium was added, and the cells were kept in culture and undisturbed for 12-14 days, during which time the surviving cells spawned a colony of proliferating cells. Colonies were visualized by staining for 4 hours with $1 \%$ methylene blue (in methanol) and then counted. For the CFAs, the columns represent the mean percentage of surviving cells from triplicate experiments; bars indicate the SD. The number of colonies obtained from the untreated controls was set at $100 \%$. The asterisk indicates significance $(p<0.05)$. 
A

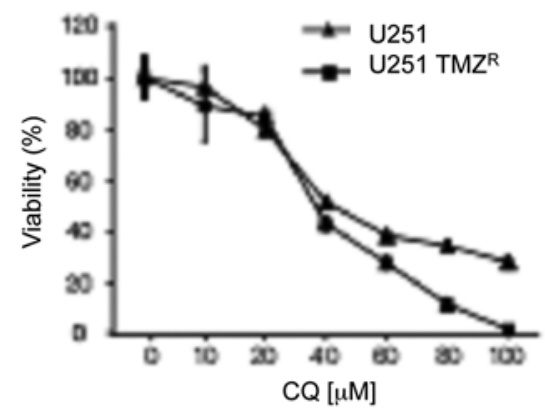

B

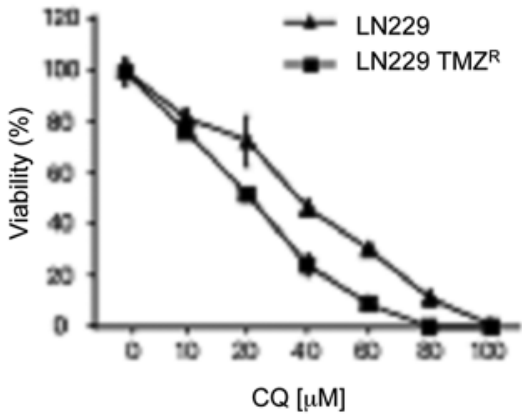

C

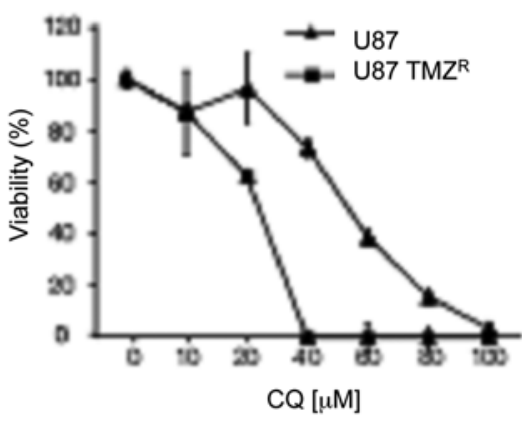

D

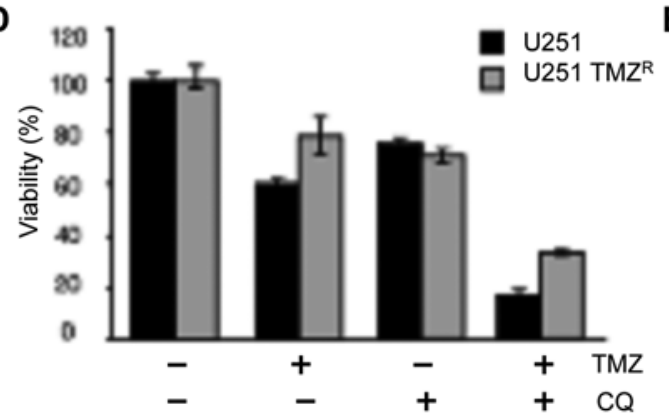

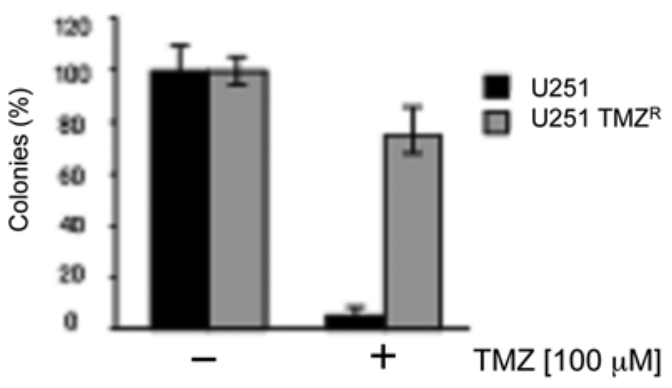
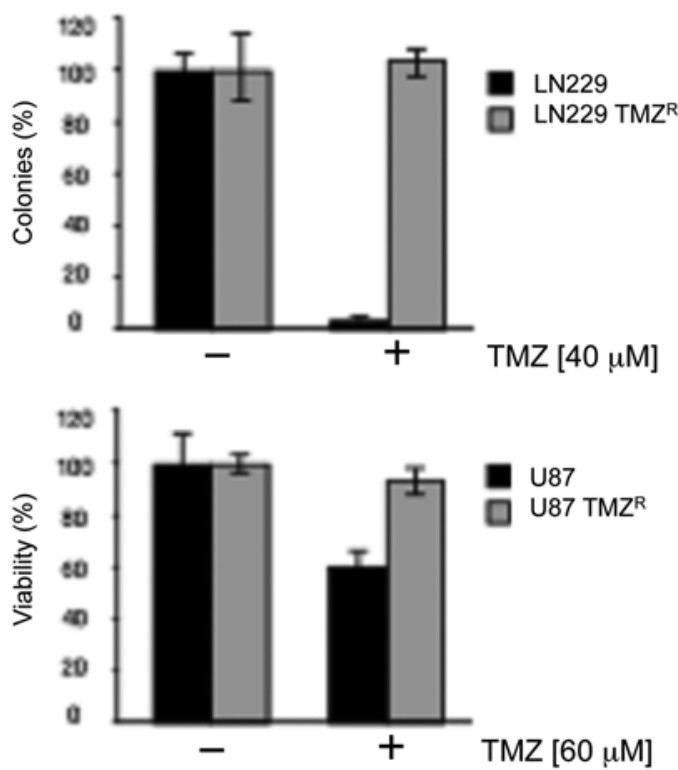

E

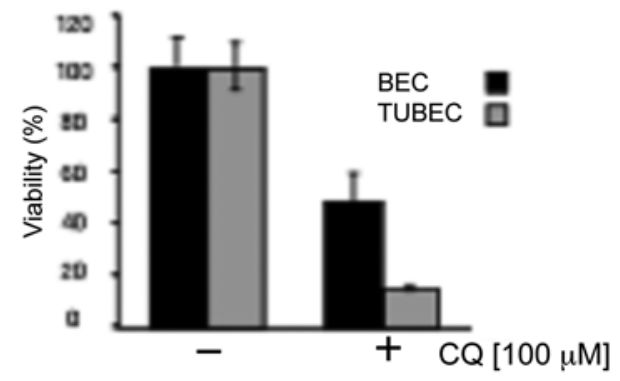

FIG. 4. Chloroquine is more toxic to $T M Z^{R}$ tumor cells. Cell growth and survival of various $T_{M} Z^{S}$ and $T M Z^{R}$ cell lines were determined by MTT assays after 48 hours of culture in the presence of increasing concentrations of CQ (A-C [left] and D). Because U87 cells do not form colonies, they were treated with TMZ in a long-term MTT assay (C [right]). Each treatment condition was set up in quadruplicate, and each experiment was repeated 1-5 times independently; bars indicate the SD. In a series of CFAs, cells were either untreated or treated with TMZ for 48 hours ( $\mathbf{A}$ and $\mathbf{B}[$ right]). Subsequently, the drug was removed, fresh growth medium was added, and the cells were kept in culture and undisturbed for 12-14 days, during which time the surviving cells spawned a colony of proliferating cells. Colonies were visualized by staining for 4 hours with $1 \%$ methylene blue (in methanol) and then counted. For the CFAs, the columns represent the mean percentage of surviving cells from triplicate experiments; bars indicate the SD. The number of colonies obtained from the untreated controls was set at $100 \%$. Normal BECs and TuBECs were treated with $C Q$ for 48 hours in the MTT assay (E).

$\mathrm{TMZ}^{\mathrm{R}}$ glioma cell lines in a series of MTT assays with $\mathrm{CQ}$, we observed that $\mathrm{CQ}$ killed $\mathrm{TMZ}^{\mathrm{R}}$ cell lines more effectively than their parental $\mathrm{TMZ}^{\mathrm{S}}$ cell lines (Fig. $4 \mathrm{~A}-\mathrm{C}$, left). For example, $100 \mu \mathrm{M} C \mathrm{CQ}$ reduced the percent viability of U251-TMZ glioma cells by $\sim 70 \%$. However,
$100 \mu \mathrm{M}$ CQ reduced the percent viability of U251-TMZ ${ }^{\mathrm{R}}$ glioma cells by $\sim 100 \%$. In addition, $40 \mu \mathrm{M}$ CQ reduced the percent viability of LN229-TMZ ${ }^{\mathrm{S}}$ glioma cells by $50 \%$. In contrast, $40 \mu \mathrm{M} C Q$ reduced the percent viability of LN229-TMZ ${ }^{\mathrm{R}}$ glioma cells by $75 \%$. Last, $40 \mu \mathrm{M}$ CQ 
reduced the percent viability of U87-TMZ ${ }^{\text {s }}$ glioma cells (p53 wild type, PTEN mutant) by $50 \%$. Nevertheless, 40 $\mu \mathrm{M}$ CQ completely eliminated the U87-TMZR glioma cells.

To confirm $\mathrm{TMZ}$ resistance in our $\mathrm{TMZ}^{\mathrm{R}}$ glioma cells in comparison with their parental $\mathrm{TMZ}^{\mathrm{S}}$ glioma cells, we treated $\mathrm{TMZ}^{\mathrm{S}}$ and $\mathrm{TMZ}^{\mathrm{R}}$ glioma cells in a series of long-term cell-viability assays (Fig. 4A-C, right). In a series of CFAs, we observed that $100 \mu \mathrm{M}$ and $40 \mu \mathrm{M}$ of TMZ reduced the percentage of colonies formed in U251-TMZS and LN229-TMZs glioma cells, respectively, by $\sim 95 \%$. However, $100 \mu \mathrm{M}$ and $40 \mu \mathrm{M}$ TMZ reduced the percentage of colonies formed in U251-TMZ ${ }^{\mathrm{R}}$ and LN229-TMZR ${ }^{\mathrm{R}}$ glioma cells, respectively, by $\sim 20 \%$ and $0 \%$. Because U87 does not form colonies well enough for a CFA, we determined the cell viability of U87-TMZ ${ }^{\mathrm{S}}$ and $\mathrm{U} 7-\mathrm{TMZ}^{\mathrm{R}}$ glioma cells via a long-term MTT assay (Fig. $4 \mathrm{C}$, right). TMZ (at $60 \mu \mathrm{M}$ ) reduced the percent viability of U87-TMZ ${ }^{\mathrm{R}}$ glioma cells by $\sim 40 \%$; however, it reduced the percent viability of U87-TMZS cells by $\sim 5 \%$.

To investigate whether CQ can maintain its ability to enhance $\mathrm{TMZ}$ cytotoxicity in $\mathrm{TMZ}^{\mathrm{R}}$ cells, we treated U251-TMZ ${ }^{\mathrm{R}}$ cells with TMZ in the presence or absence of CQ (Fig. 4D). We found that CQ enhanced the cytotoxic effects of TMZ in U251-TMZ ${ }^{\mathrm{R}}$ cells in a manner similar to that of its parental U251-TMZs cell line. TMZ $(20 \mu \mathrm{M})$ reduced the percentage of colonies formed in U251-TMZS cells by $\sim 40 \%$; furthermore, $100 \mu \mathrm{M}$ TMZ reduced the percentage of colonies formed in U251$\mathrm{TMZ}^{\mathrm{R}}$ cells by $\sim 20 \%$. CQ $(10 \mu \mathrm{M})$, on the other hand, reduced the percentage of colonies formed in both U251TMZ $^{\mathrm{S}}$ and U251-TMZR cells by $\sim 20 \%$. However, $20 \mu \mathrm{M}$ TMZ combined with $10 \mu \mathrm{M} C Q$ reduced the percentage of colonies formed in U251-TMZ ${ }^{\text {s }}$ cells by $\sim 80 \%$. In addition, $100 \mu \mathrm{M}$ TMZ combined with $10 \mu \mathrm{M}$ CQ reduced the percentage of colonies formed in U251-TMZ ${ }^{\mathrm{R}}$ cells by $\sim 60 \%$. The combined regimen of CQ and TMZ displayed calculated combination indices in U251-TMZ ${ }^{\mathrm{S}}$ and U251$\mathrm{TMZ}^{\mathrm{R}}$ cells of $<1$, indicating synergism between the two chemotherapies.

In addition to determining CQ cytotoxicity in $\mathrm{TMZ}^{\mathrm{R}}$ glioma cells, we investigated CQ cytotoxicity in $\mathrm{TMZ}{ }^{\mathrm{R}}$ TuBECs. We previously reported that TuBECs resist the cytotoxic effects of TMZ, when compared with normal BECs, and that knockdown of GRP78 in TuBECs chemosensitizes TuBECs to TMZ. ${ }^{42}$ It is interesting to note that in a series of MTT assays, we observed that CQ killed $\mathrm{TMZ}^{\mathrm{R}}$ TuBECs more efficiently than TMZ ${ }^{\mathrm{S}}$ BECs (Fig. $4 \mathrm{E})$. Taken together, these data indicate that $\mathrm{CQ}$ killed the $\mathrm{TMZ}^{\mathrm{R}}$ cell lines $\left(\mathrm{U} 251-\mathrm{TMZ}^{\mathrm{R}}\right.$, LN229-TMZ ${ }^{\mathrm{R}}$, U87$\mathrm{TMZ}^{\mathrm{R}}$, and TuBECs) better than their parental $\mathrm{TMZ}^{\mathrm{S}}$ cell lines (U251-TMZ ${ }^{\mathrm{s}}$, LN229-TMZs ${ }^{\mathrm{s}}$, U87-TMZ ${ }^{\mathrm{s}}$, and $\mathrm{BECs})$. Thus, $\mathrm{TMZ}^{\mathrm{R}}$ cells may be more sensitive to $\mathrm{CQ}$.

Chloroquine Enhances the Cytotoxic Effects of Temozolomide by Blocking Autophagy

Because CQ enhanced the cytotoxic effects of TMZ in a series of Western blot assays and CFAs, we set out to confirm our findings by investigating the combined effects of TMZ and CQ in vitro and in vivo. In a Western blot assay, blocking autophagy clearance with $25 \mu \mathrm{M}$
$\mathrm{CQ}$ resulted in a modest increase in the levels of $\mathrm{LC} 3 \mathrm{~B}-$ II (LC3B-I/LC3B-II $\approx 1)$, ubiquitinated proteins, $\mathrm{CHOP} /$ GADD-153, and cleaved PARP in U251 glioma cells (Fig. 5 upper). However, after blocking autophagy clearance with $25 \mu \mathrm{M}$ CQ, $100 \mu \mathrm{M}$ TMZ significantly increased the
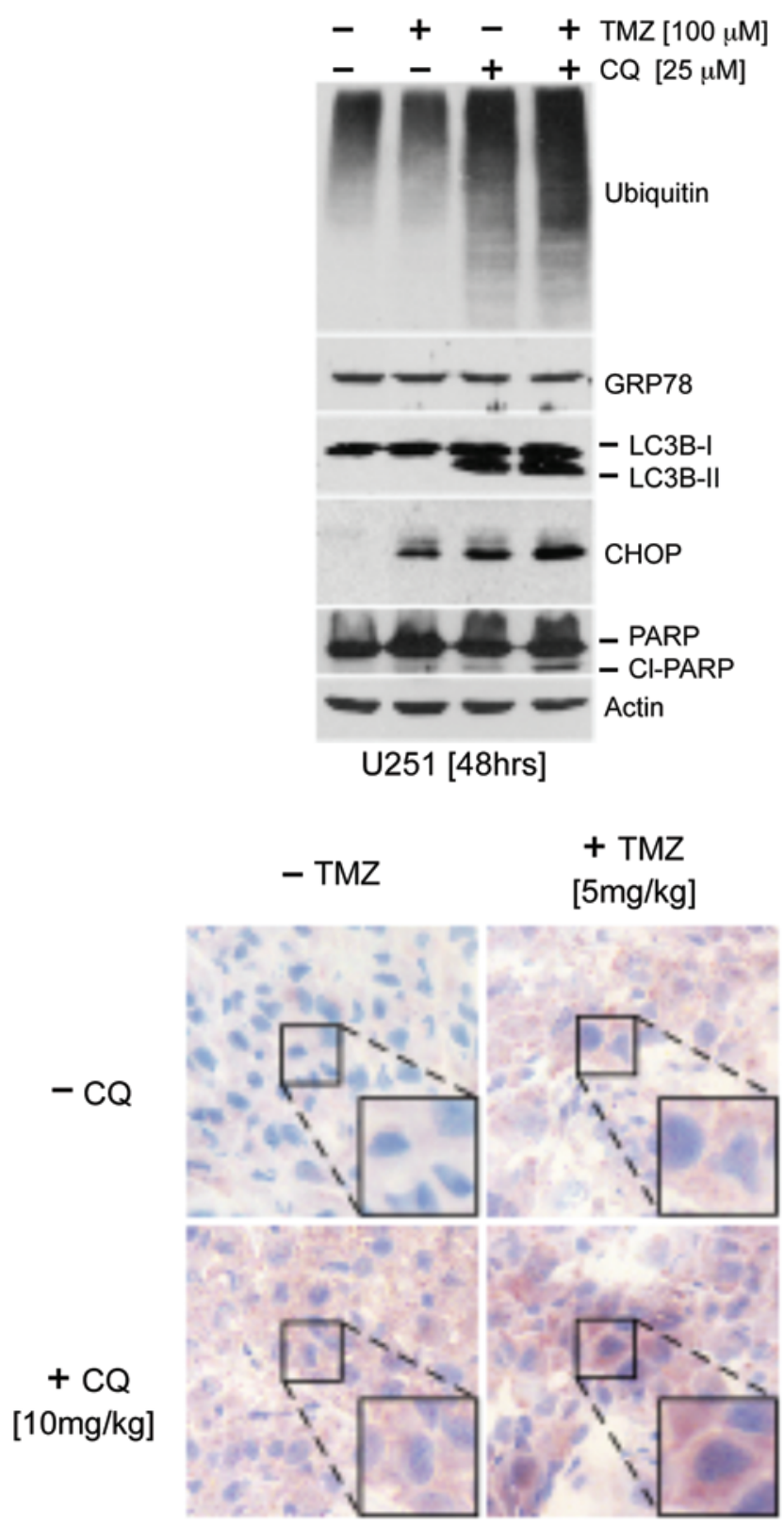

FIG. 5. Chloroquine enhances the cytotoxic effects of TMZ in vitro and in vivo. U251 cells were seeded for Western blot analysis and were either untreated or treated with $25 \mu \mathrm{M} \mathrm{CQ}, 100 \mu \mathrm{M}$ TMZ individually, or in combination for 48 hours. Upper: Expression levels of polyubiquitinated proteins, GRP78, CHOP/GADD-153, LC3B-I/II, and cleaved PARP, with antibodies specifically directed to each protein, were analyzed. $\beta$-Actin (Actin) was used as a loading control. Tumor-bearing mice were either untreated or treated with $10 \mathrm{mg} / \mathrm{kg} \mathrm{CQ}$ and $5 \mathrm{mg} / \mathrm{kg}$ TMZ individually or in combination. Lower: Fifty hours later, the animals were killed, and the tumors were analyzed by immunohistochemical staining for CHOP (ER stress indicator). Tumor tissue stained with $\mathrm{CHOP}$ antibodies (left). Enlarged areas of the sections indicated by the small rectangles (right). 
levels of LC3B-II (LC3B-I/LC3B-II < 1), ubiquitinated proteins, CHOP/GADD-153, and cleaved PARP. These results suggest that TMZ stimulates autophagy; however, when TMZ is in the presence of CQ, TMZ causes greater increases in autophagosome accumulation and apoptosis than CQ alone.

Apart from investigating the combined cytotoxic effects of CQ and TMZ in a Western blot assay, we set out to investigate the combined cytotoxic effects of CQ and TMZ in vivo (Fig. 5 lower). We implanted nude mice subcutaneously with U87 glioma cells and allowed the implanted cells to form tumors. After sizable tumors formed, we randomly assigned the mice before treating them via gavage for 48 hours with water, $10 \mathrm{mg} / \mathrm{kg} \mathrm{CQ}, 5$ $\mathrm{mg} / \mathrm{kg} \mathrm{TMZ}$, or the combination of CQ and TMZ. After harvesting and immunostaining the tumors for $\mathrm{CHOP} /$ GADD-153, tumors treated with CQ or TMZ alone demonstrated a modest induction of CHOP/GADD-153 in comparison with the untreated control. However, tumors treated with CQ in combination with TMZ displayed higher levels of $\mathrm{CHOP} / \mathrm{GADD}-153$ expression than the tumors treated with CQ or TMZ alone. Altogether, our results demonstrate that blocking autophagy with CQ enhances the cytotoxic effects of TMZ in vitro and in vivo.

\section{Discussion}

Glioblastoma multiforme is a highly malignant brain tumor that is difficult to treat and carries a very poor prognosis. Conventional therapies for patients with newly diagnosed GBM include surgical tumor resection followed by radiation therapy and chemotherapy with the DNAalkylating agent TMZ. In a recent study, the quinolinebased antimalarial, $\mathrm{CQ}$, in combination with conventional therapy significantly increased the mean survival probability of patients with GBM from 11.4 to 25 months. ${ }^{4}$ The authors of that study attributed the antimutagenic properties of CQ to its ability to enhance TMZ. ${ }^{5}$ They hypothesized that mutagenic chemotherapies, such as TMZ and carmustine, cause hypermutations in glioma cell clones that eventually lead to drug resistance. Moreover, they speculated that adding CQ would reduce TMZ-induced hypermutation in glioma cells and prevent the development of $\mathrm{TMZ} \mathrm{Z}^{\mathrm{R}}$ tumor cells. However, alternative studies have suggested that hypermethylation of the promoter for the DNA repair enzyme $\mathrm{O}^{6}$-methylguanine-DNA methyltransferase ( $\left.\mathrm{O}^{6}-\mathrm{MGMT}\right)$ and the loss of DNA repair are positive predictors for patients who will respond to TMZ therapy. ${ }^{14,31,34}$ Thus, the mechanistic role of CQ as an enhancer of TMZ remains unclear.

Temozolomide was previously reported to have an effect on the autophagy system. ${ }^{18}$ Nevertheless, recent evidence brought into question whether autophagy is involved in tumor progression or tumor suppression..$^{16,19,24,26,29} \mathrm{With}$ respect to the relationship between TMZ and autophagy, TMZ treatment of GFP-LC3-overexpressing cells resulted in an increase in autophagosome formation. ${ }^{18}$ However, bafilomycin A, a vesicular type proton-pump inhibitor that prevents the maintenance of the lysosomal acidic environment and inhibits autophagy clearance, enhanced TMZ cytotoxicity in glioma cells. ${ }^{18}$ Because CQ accumulates in lysosomes, inhibits autophagy clearance, and is a clinically useful enhancer of TMZ, we set out to determine whether CQ enhances the cytotoxic effects of TMZ in a manner similar to that of bafilomycin A.

When we treated glioma cells with CQ, we found that CQ blocked autophagy while enhancing the cytotoxic effects of TMZ. First, we discovered that knockdown of GRP78 mitigated the cytotoxic effects of CQ but enhanced the cytotoxic effects of TMZ. We demonstrated that GRP78-dependent autophagy, but not BECN1dependent autophagy, limited the cytotoxic effects of TMZ (Fig. 1B). Next, we found that CQ efficiently killed $\mathrm{TMZ}^{\mathrm{R}}$ cells, including U251-TMZ ${ }^{\mathrm{R}}$, LN229-TMZ ${ }^{\mathrm{R}}$, and U87-TMZ $^{\mathrm{R}}$ cells and TuBECs. Furthermore, we showed that CQ was able to block GRP78-dependent autophagy in addition to PI3KC3-BECN1-dependent autophagy. Thus, when we combined CQ with TMZ, CQ synergistically enhanced the cytotoxic effects of TMZ by blocking autophagy, which led to an increase in autophagosome formation, ER stress, and apoptosis in vitro and in vivo. This mechanism is represented schematically in Fig. 6.

The implications for establishing the mechanism of the combined effects of CQ and TMZ are 3-fold. First of all, we demonstrated that GRP78-dependent autophagy limited the cytotoxic effects of TMZ. GRP78 confers chemoresistance to several tumor types and may do so via its association with autophagosome formation. ${ }^{6,7,10,25,33,35,42}$ Thus, blocking GRP78-dependent autophagy with CQ may chemosensitize various types of tumor cells and chemosensitize glioma cells to TMZ. Second, basal levels of GRP78-dependent autophagy may be a predictor for CQ sensitivity and TMZ resistance in tumor cells. Last, because we established that CQ blocks GRP78-dependent autophagy while enhancing the cytotoxic effects of TMZ, the development of alternative and/or specific inhibitors of GRP78-dependent autophagy may prove to be even more useful in enhancing the cytotoxic effects of TMZ in the clinic.

\section{Conclusions}

Our data provide a basis for the use of CQ in conjunction with TMZ. The clinical protocol for the administration of CQ in combination with TMZ is the subject of ongoing clinical trials. In particular, whether CQ should be administered to TMZ as part of the traditional highdose TMZ regimen or the low-dose metronomic TMZ regimen will need to be explored. The ability of $C Q$ to induce apoptosis in $\mathrm{TMZ}^{\mathrm{R}}$ cell lines is particularly encouraging and suggests that it may be used to extend the response for patients who have progressed on TMZ.

\section{Disclosure}

The authors report no conflict of interest concerning the materials or methods used in this study or the findings specified in this paper.

Author contributions to the study and manuscript preparation include the following. Conception and design: Chen, Golden, Hofman, Schönthal, Cho. Acquisition of data: Golden, Cho, Louie. Analysis and interpretation of data: all authors. Drafting the article: Chen, Golden, Cho, Hofman, Schönthal. Critically revising the 


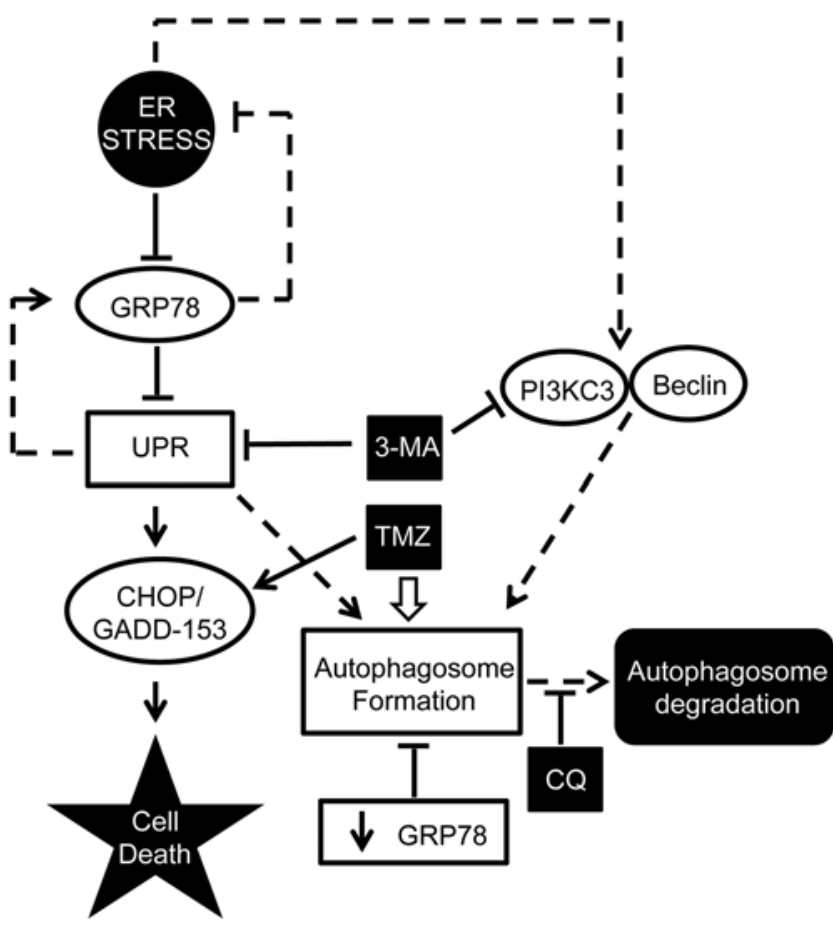

FIG. 6. Schematic diagram. CQ blocks autophagy while enhancing TMZ cytotoxicity. Tumor cells have developed adaptive mechanisms for dealing with chronic ER stress. Chronic ER stress caused by misfolded proteins and nutrient depletion activates BECN1-PI3KC3-dependent autophagy and the UPR cytoprotective pathways (dashed lines). A chronically activated UPR induces the production of the cytoprotective protein GRP78 and the formation of autophagosomes. Autophagosomes envelope polyubiquitinated ER protein aggregates for eventual lysosomal degradation, thereby reducing the levels of misfolded proteins and mitigating ER stress while providing for essential building blocks necessary for the biosynthesis of complex cellular molecules. In contrast to chronic ER stress, acute ER stress results in high levels of misfolded proteins, which rapidly accumulate in the ER, sequester GRP78, and completely block GRP78 from suppressing UPR transducers. A complete loss of UPR transducer suppression triggers the induction of the ER stress proapoptotic protein CHOP/GADD-153. CQ is a lysosomotrope that exerts its cytotoxic effects by blocking autophagosome degradation and activating induction of CHOP/GADD-153. Knockdown of BECN1 or GRP78 or treatment with the PI3KC3 and UPR inhibitor, 3-MA, blocks autophagosome formation and reduces $\mathrm{CQ}$ cytotoxicity. TMZ exerts its cytotoxic effects by inducing CHOP/ GADD-153. However, TMZ also induces autophagosome formation, which acts to protect the cells from TMZ cytotoxicity (open arrow). Knockdown of GRP78 (but not BECN1) or treatment with 3-MA or CQ blocks autophagy and enhances TMZ cytotoxicity.

article: Chen, Hofman. Statistical analysis: Louie. Study supervision: Chen, Hofman, Schönthal.

\section{References}

1. Araki N, Hamasaki M, Egami Y, Hatae T: Effect of 3-methyladenine on the fusion process of macropinosomes in EGFstimulated A431 cells. Cell Struct Funct 31:145-157, 2006

2. Bernales S, McDonald KL, Walter P: Autophagy counterbalances endoplasmic reticulum expansion during the unfolded protein response. PLoS Biol 4:e423, 2006

3. Bobola MS, Tseng SH, Blank A, Berger MS, Silber JR: Role of O6-methylguanine-DNA methyltransferase in resistance of human brain tumor cell lines to the clinically relevant meth- ylating agents temozolomide and streptozotocin. Clin Cancer Res 2:735-741, 1996

4. Briceño E, Calderon A, Sotelo J: Institutional experience with chloroquine as an adjuvant to the therapy for glioblastoma multiforme. Surg Neurol 67:388-391, 2007

5. Briceño E, Reyes S, Sotelo J: Therapy of glioblastoma multiforme improved by the antimutagenic chloroquine. Neurosurg Focus 14(2):E3, 2003

6. Carew JS, Nawrocki ST, Cleveland JL: Modulating autophagy for therapeutic benefit. Autophagy 3:464-467, 2007

7. Carew JS, Nawrocki ST, Kahue CN, Zhang H, Yang C, Chung L, et al: Targeting autophagy augments the anticancer activity of the histone deacetylase inhibitor SAHA to overcome Bcr-Abl-mediated drug resistance. Blood 110:313-322, 2007

8. Codogno P, Meijer AJ: Autophagy and signaling: their role in cell survival and cell death. Cell Death Differ 12 (Suppl 2):1509-1518, 2005

9. Dang CV: Antimalarial therapy prevents Myc-induced lymphoma. J Clin Invest 118:15-17, 2008

10. Dong D, Ni M, Li J, Xiong S, Ye W, Virrey JJ, et al: Critical role of the stress chaperone GRP78/BiP in tumor proliferation, survival, and tumor angiogenesis in transgene-induced mammary tumor development. Cancer Res 68:498-505, 2008

11. Ermakova SP, Kang BS, Choi BY, Choi HS, Schuster TF, Ma WY, et al: (-)-Epigallocatechin gallate overcomes resistance to etoposide-induced cell death by targeting the molecular chaperone glucose-regulated protein 78. Cancer Res 66:9260-9269, 2006

12. Esteller M, Garcia-Foncillas J, Andion E, Goodman SN, Hidalgo OF, Vanaclocha V, et al: Inactivation of the DNArepair gene MGMT and the clinical response of gliomas to alkylating agents. N Engl J Med 343:1350-1354, 2000

13. Fontijn D, Adema AD, Bhakat KK, Pinedo HM, Peters GJ, Boven E: O6-methylguanine-DNA-methyltransferase promoter demethylation is involved in basic fibroblast growth factor induced resistance against temozolomide in human melanoma cells. Mol Cancer Ther 6:2807-2815, 2007

14. Hegi ME, Diserens AC, Gorlia T, Hamou MF, de Tribolet N, Weller M, et al: MGMT gene silencing and benefit from temozolomide in glioblastoma. N Engl J Med 352:997-1003, 2005

15. Høyer-Hansen M, Jäättelä M: Connecting endoplasmic reticulum stress to autophagy by unfolded protein response and calcium. Cell Death Differ 14:1576-1582, 2007

16. Jin S, White E: Role of autophagy in cancer: management of metabolic stress. Autophagy 3:28-31, 2007

17. Kang KB, Zhu C, Yong SK, Gao Q, Wong MC: Enhanced sensitivity of celecoxib in human glioblastoma cells: Induction of DNA damage leading to p53-dependent G1 cell cycle arrest and autophagy. Mol Cancer 8:66, 2009

18. Kanzawa T, Germano IM, Komata T, Ito H, Kondo Y, Kondo S: Role of autophagy in temozolomide-induced cytotoxicity for malignant glioma cells. Cell Death Differ 11:448-457, 2004

19. Karantza-Wadsworth V, White E: Role of autophagy in breast cancer. Autophagy 3:610-613, 2007

20. Kardosh A, Golden EB, Pyrko P, Uddin J, Hofman FM, Chen TC, et al: Aggravated endoplasmic reticulum stress as a basis for enhanced glioblastoma cell killing by bortezomib in combination with celecoxib or its non-coxib analogue, 2,5-dimethyl-celecoxib. Cancer Res 68:843-851, 2008

21. Kardosh A, Soriano N, Liu YT, Uddin J, Petasis NA, Hofman FM, et al: Multitarget inhibition of drug-resistant multiple myeloma cell lines by dimethyl-celecoxib (DMC), a non-COX-2 inhibitory analog of celecoxib. Blood 106:4330-4338, 2005

22. Klionsky DJ, Abeliovich H, Agostinis P, Agrawal DK, Aliev G, Askew DS, et al: Guidelines for the use and interpretation of assays for monitoring autophagy in higher eukaryotes. Autophagy 4:151-175, 2008 
23. Kokkinakis DM, Hoffman RM, Frenkel EP, Wick JB, Han Q, $\mathrm{Xu}$ M, et al: Synergy between methionine stress and chemotherapy in the treatment of brain tumor xenografts in athymic mice. Cancer Res 61:4017-4023, 2001

24. Kondo Y, Kanzawa T, Sawaya R, Kondo S: The role of autophagy in cancer development and response to therapy. Nat Rev Cancer 5:726-734, 2005

25. Lee E, Nichols P, Spicer D, Groshen S, Yu MC, Lee AS: GRP78 as a novel predictor of responsiveness to chemotherapy in breast cancer. Cancer Res 66:7849-7853, 2006

26. Levine B: Unraveling the role of autophagy in cancer. Autophagy 2:65-66, 2006

27. Li J, Ni M, Lee B, Barron E, Hinton DR, Lee AS: The unfolded protein response regulator GRP78/BiP is required for endoplasmic reticulum integrity and stress-induced autophagy in mammalian cells. Cell Death Differ 15:1460-1471, 2008

28. Marx J: Autophagy: is it cancer's friend or foe? Science 312:1160-1161, 2006

29. Mathew R, Karantza-Wadsworth V, White E: Role of autophagy in cancer. Nat Rev Cancer 7:961-967, 2007

30. Mizushima $\mathbf{N}$ : Methods for monitoring autophagy. Int J Biochem Cell Biol 36:2491-2502, 2004

31. Paz MF, Yaya-Tur R, Rojas-Marcos I, Reynes G, Pollan M, Aguirre-Cruz L, et al: $\mathrm{CpG}$ island hypermethylation of the DNA repair enzyme methyltransferase predicts response to temozolomide in primary gliomas. Clin Cancer Res 10:49334938, 2004

32. Pyrko P, Kardosh A, Liu YT, Soriano N, Xiong W, Chow RH, et al: Calcium-activated endoplasmic reticulum stress as a major component of tumor cell death induced by 2,5-dimethylcelecoxib, a non-coxib analogue of celecoxib. Mol Cancer Ther 6:1262-1275, 2007

33. Pyrko P, Schönthal AH, Hofman FM, Chen TC, Lee AS: The unfolded protein response regulator GRP78/BiP as a novel target for increasing chemosensitivity in malignant gliomas. Cancer Res 67:9809-9816, 2007

34. Sasai K, Akagi T, Aoyanagi E, Tabu K, Kaneko S, Tanaka S: O6-methylguanine-DNA methyltransferase is downregulated in transformed astrocyte cells: implications for anti-glioma therapies. Mol Cancer 6:36, 2007

35. Schönthal AH: Endoplasmic reticulum stress and autophagy as targets for cancer therapy. Cancer Lett 275:163-169, 2009

36. Shingu T, Yamada K, Hara N, Moritake K, Osago H, Terashima $\mathrm{M}$, et al: Growth inhibition of human malignant glioma cells induced by the PI3-K-specific inhibitor. J Neurosurg 98:154161,2003

37. Shono T, Tofilon PJ, Schaefer TS, Parikh D, Liu TJ, Lang FF: Apoptosis induced by adenovirus-mediated p53 gene transfer in human glioma correlates with site-specific phosphorylation. Cancer Res 62:1069-1076, 2002

38. Slater AF, Cerami A: Inhibition by chloroquine of a novel haem polymerase enzyme activity in malaria trophozoites. Nature 355:167-169, 1992

39. Sotelo J, Briceño E, López-González MA: Adding chloroquine to conventional treatment for glioblastoma multiforme: a randomized, double-blind, placebo-controlled trial. Ann Intern Med 144:337-343, 2006

40. Taverna P, Catapano CV, Citti L, Bonfanti M, D'Incalci M: Influence of $\mathrm{O}^{6}$-methylguanine on DNA damage and cytotoxicity of temozolomide in L1210 mouse leukemia sensitive and resistant to chloroethylnitrosoureas. Anticancer Drugs 3:401405, 1992

41. Tentori L, Orlando L, Lacal PM, Benincasa E, Faraoni I, Bonmassar $\mathrm{E}$, et al: Inhibition of $\mathrm{O}^{6}$-alkylguanine DNAalkyltransferase or poly(ADP-ribose) polymerase increases susceptibility of leukemic cells to apoptosis induced by temozolomide. Mol Pharmacol 52:249-258, 1997

42. Virrey JJ, Dong D, Stiles C, Patterson JB, Pen L, Ni M, et al: Stress chaperone GRP78/BiP confers chemoresistance to tumor-associated endothelial cells. Mol Cancer Res 6:12681275,2008

43. Yorimitsu T, Nair U, Yang Z, Klionsky DJ: Endoplasmic reticulum stress triggers autophagy. J Biol Chem 281:3029930304, 2006

44. Zhao L, Wientjes MG, Au JL: Evaluation of combination chemotherapy: integration of nonlinear regression, curve shift, isobologram, and combination index analyses. Clin Cancer Res 10:7994-8004, 2004

Manuscript submitted August 15, 2014.

Accepted September 26, 2014.

Please include this information when citing this paper: DOI: 10.3171/2014.9.FOCUS14504.

Address correspondence to: Thomas C. Chen, M.D., Ph.D., Departments of Neurosurgery and Pathology, University of Southern California, 2011 Zonal Ave., Los Angeles, CA 90033. email: thomas.chen@medmail.usc.edu. 